\title{
Neural Connectivity: How to Reinforce the Bidirectional Synapse Between Basic Neuroscience and Routine Neurosurgical Practice?
}

\author{
Hugues Duffau ${ }^{1,2 *}$ \\ ${ }^{1}$ Department of Neurosurgery, Gui de Chauliac Hospital, Montpellier University Medical Center, Montpellier, France, ${ }^{2}$ Team \\ "Plasticity of Central Nervous System, Stem Cells and Glial Tumors," National Institute for Health and Medical Research \\ (INSERM), U1191 Laboratory, Institute of Functional Genomics, University of Montpellier, Montpellier, France
}

Keywords: awake neurosurgery, brain mapping, neural connectivity, neuroscience, neuroplasticity

\section{INTRODUCTION}

The quest for all neuroscientists is to better understand neural foundations underpinning human behavior. Neurosurgery offers a unique opportunity to be directly connected to the human connectome, and to provide further findings into brain processes-complementary to current investigations mainly relying on functional neuroimaging (FNI), comprising functional MRI, and diffusion tensor imaging (DTI) (1-4). Especially, awake patients with electrostimulation mapping (ESM) may benefit from an extensive neuropsychological assessment in real-time throughout surgery. This paradigm resulted in the description of the human homunculus by Penfield and Boldrey (5) almost one century ago and in the re-visitation of cortical organization of language by Ojemann (6). However, despite these pioneering works, the contribution of neurosurgery to fundamental neurosciences remains relatively modest, particularly in comparison with the numerous FNI reports $(7,8)$. Reversely, advances in brain connectomics, which led to new propose-built neurobiological models underlying neurocognition thanks to an improved knowledge of neural connectivity, have not (yet) extensively been incorporated in neurosurgical practice.

Here, the purpose is to consider solutions to reinforce the synapse, which should be bidirectional, between basic neuroscience and routine neurosurgery, in order to bring about synergies across research and clinical worlds based upon a dual vision of scientists and physicians.

\section{CONTRIBUTION OF BRAIN SURGICAL MAPPING TO INVESTIGATE NEURAL CONNECTIVITY}

In the emerging field of connectomics, which aims at exploring neural connectivity, brain ESM during awake surgery provides direct insights into the function of both cortical structures and white matter tracts (WMT). Indeed, axonal ESM of the subcortical fibers may elicit a transient disruption of the functional network sub-served by the bundle stimulated, and consequently, may generate a specific behavioral deficit which immediately resolves when ESM stops (9). Beyond a transitory dys-synchronization within a discrete critical circuit, ESM can also disrupt between-network inter-communication, resulting in multi-tasking disorders, e.g., the patient is still able to move or to speak separately, but cannot do both simultaneously (10). Such original anatomo-functional correlations gained from on-line intraoperative corticosubcortical ESM led researchers to re-visit the functional connectivity mediating neural systems, such as movement execution and control (11), oral and written language $(12,13)$, semantics (14), executive control (15), self-evaluation (16), or theory of mind (17, 18). 
Interestingly, this ESM permitted a reappraisal of the model of the human connectome (19), and proposed a new theory relying on a meta-network (network of networks) organization of the brain, i.e., with perpetual changes of intra- and across circuit interactions allowing adapted behavior (20).

Moreover, longitudinal ESM explorations, particularly based upon serial mapping in patients who underwent several awake surgeries because of tumor relapse (21), enabled researchers to better understand mechanisms sustaining neuroplasticity (22). Remarkably, optimal recovery following massive brain resections in tumor patients, with normal scores on objective neuropsychological assessments of conation, cognition, and emotion despite surgery within structures classically deemed "eloquent" according to localizationist dogma, evidenced a considerable potential of neural configuration, higher than previously thought $(23,24)$. Nonetheless, the limitations of this plastic potential have also been demonstrated, by emphasizing the critical role of the subcortical connectivity (25). Thus, a "minimal common brain" has been suggested, with a low level of inter-individual variability and a low power of functional compensation after cerebral injury (26): such a "cerebral skeleton" is mainly constituted by WMT (27).

Neurosurgery represents a gold mine to develop innovative models in cognitive neurosciences, thanks to the direct information about neural connectivity collected into the operating room (OR) by means of ESM (19). It is regrettable that these original data which challenged the obsolete model of localizationism, e.g., by evidencing that Broca's area was non-critical for speech, were neglected for a long time by neurologists and neuroscientists (28). A solution to reunify learned societies can be to combine findings provided by ESM with those gained from FNI in healthy volunteers as well as before and after surgery in brain-damaged patients (22). Based upon different backgrounds and complementary areas of expertise (neuroanatomy, neuroimaging, awake brain mapping, cognitive neuroscience, neurophysiology, neurocomputational modeling, etc.), speaking a universal language may allow researchers to understand more rapidly and accurately neurobiology-sustaining complex human behavior. Reversely, an improved knowledge of brain circuitry could be helpful in neurosurgical practice, to optimize postoperative outcomes.

\section{HOW TO INTEGRATE A BETTER UNDERSTANDING OF THE CEREBRAL CONNECTOME INTO NEUROSURGICAL PRACTICE?}

For many decades, neurosurgeons mainly paid attention to the cortex, with few considerations regarding subcortical connectivity. Beyond research purposes, recent advances in DTI resulted in an improved investigation of WMT for surgical planning $(29,30)$. Moreover, preoperative tractography data were incorporated into neuronavigational systems to better define surgical approach and limits of resection into the $\operatorname{OR}(31,32)$. However, even though these technological developments played a major role in basic research to explore the connectome (3,
8), and started to bring the gap between neuroscience and clinical applications, the link across both worlds is still weak and superficial. Indeed, the majority of neurosurgeons has no background in FNI methodology and has the wrong belief that DTI is a reliable insight into WMT function. Yet, despite a growing excitement in imaging-guided neurosurgery, these techniques intrinsically suffer from major limitations (from the data acquisition to the statistical models used) $(33,34)$, the main one being that tractography does not provide any information about the function of subcortical fibers-but is only an indirect reflection of their structures (35). Therefore, an abusive surgical use of these methods whose pitfalls are poorly controlled, even if initially designed to help neurosurgeons, may paradoxically become dangerous. For example, FNI may result in a nonselection for surgery while the tumor could have been removed (with a loss of chance from an oncological perspective), or conversely, may lead to the resection being pushed too far by cutting critical pathways not identified as essential by DTI (with some loss likely from a functional perspective) (36). Furthermore, although FNI is a fantastic didactic tool to help junior neurosurgeons to build an accurate $3 \mathrm{D}$ representation of structural and functional connectivity in their own mental imagery, especially when combined with dissection in specimens $(37,38)$, the overuse of this technology for brain surgery may lead to the opposite effect, i.e., to an addiction to FNI, preventing an optimal surgical act if this tool was unavailable (36).

To reinforce the synapse with fundamental research, neurosurgeons should be neuroscientists, since he/she also has the responsibility to explore the connectome by him/herself, with the ultimate goal of improving postoperative outcomes. Thus, besides a better comprehension of the potentials and drawbacks of FNI for its more appropriate utilization, intraoperative ESM should be used more systematically (39). In addition to cortical mapping, axonal ESM is the sole methodology enabling a direct study of WMT function, concerning one specific bundle and interplay between neural circuits (10). Awake mapping led to a paradigmatic shift from image-guided resection to functionalguided resection, especially in neuro-oncology (40). Remarkably, awake ESM guiding the resection until the individual eloquent cortico-subcortical networks have been encountered resulted in an improvement of both neurological and oncological outcomes (41). Functionally speaking, a connectome-based resection allowed for a significant decrease of neurological morbidity (42-44) with preservation of conation, language, and highercognitive functions (e.g., complex movement control, verbal and non-verbal semantics, executive functions, mentalizing or metacognition) (45-48) making it possible to resume a normal life, including return to work in $97 \%$ of patients (49) - even for tumors involving areas presumed to be "eloquent" in a rigid localizationist framework $(23,24)$. Oncologically speaking, functional mapping-guided surgery has enabled a significant increase of extent of resection and overall survival, both in low-grade and high-grade gliomas (50-53). Yet, although ESM is now the gold standard for glioma surgery (39), it is still underused in neurosurgery in general.

This concept of connectome-based surgery represents an actual solution to introduce more robust neuroscience into the 
OR. Firstly, beyond its participation in the development of original models of neurocognition $(12,13,19,20)$, awake ESM also enabled the elaboration of a human atlas of neuroplasticity (25) and an atlas of critical cortico-subcortical networks (54-56). This can be helpful for neurosurgeons to predict whether the patient will recover or not according to the extent of resection, especially by highlighting the structures with a low potential of reorganization, such as the subcortical connectivity-the socalled "minimal common brain" mentioned above (27). To facilitate presurgical planning by predicting the distribution of essential neural connections to be preserved, a tool has recently been proposed for a practical use: it allows automated alignment of the cortico-subcortical maps of this probabilistic atlas with T1weighted MRI of a given patient (57). These data can also be used to estimate before surgery the extent of resection thanks to a probabilistic map of tumor resectability, computed on the basis of postoperative residual glioma voluntarily left because of invading critical structures identified by intraoperative ESM (58).

Secondly, these new cognitive models of meta-networking organization of brain functions $(12,13,19,20)$ and the new atlases built based upon $\operatorname{ESM}(25,27,54-57)$ represent unmatched educational tools for neurosurgeons to learn $3 \mathrm{D}$ neural connectivity. Indeed, they provide real structuralfunctional information collected intraoperatively in patients who underwent awake surgery, and not virtual data as given by FNI, with different reconstructions according to the biostatistical modeling employed.

Thirdly, besides neuro-oncology, application of the concept of connectome-based resection relying on a better understanding of dynamic interplay within and across neural networks, may be considered in other fields of brain surgery. Surprisingly, although awake ESM was initially developed in epilepsy surgery $(5,6$, 59 ), most of the series dedicated to non-tumoral epilepsy did not use intraoperative mapping in the modern literature. Yet, recent publications supported again the positive role of awake resection for epilepsy, notably with mapping of the subcortical pathways, (such as optic tracts to avoid visual field deficits), in a connectome paradigm of brain processing $(60,61)$ - knowing that the mechanisms of neuroplasticity are not similar in lesional vs. non-lesional epilepsy (62). An improved exploration of neural connectivity is also valuable for surgical approaches to deep lesions located within hard-to-reach areas. Typically, a transcortical approach to have access to the insula should take into account the sub-opercular connectivity, particularly the frontal part of the inferior fronto-occipital fasciculus (IFOF) and of the superior longitudinal fasciculus/arcuate fasciculus (AF) complex (63). Similarly, a transcortico-subcortical approach to the left posterior Medio basal region necessitates the knowledge, detection, and preservation of relevant WMT including the optic pathways, AF, IFOF, and inferior longitudinal fasciculus (64). Also, for deep cavernomas, the best surgical corridor through the subcortical fibers should be defined by validating in real-time that the neural connectivity crossed to reach the lesion was not crucial for brain functions (65). This is especially valuable for lesions involving a neural crossroad, e.g., the ventral precentral fiber intersection area (66) or the temporoparietal fiber intersection area traversed by seven WMT (67). Finally, such a 3D mental representation in the brain's neurosurgeon is also useful for emergent surgery, such as the evacuation of a left deep temporoinsular hematoma under general anesthesia, in an aphasic patient with mass effect. If the patient remained aphasic after surgery, it could be thought that this was related to an irreversible damage generated by the hematoma, whereas in some cases, it might be due to a traumatic surgical corridor through the critical fibers, such as the IFOF-meaning that the patient could have recovered if the approach would have been modified thanks to better knowledge of the connectome, even in asleep patients. To this end, fiber dissection in cadavers to accelerate the learning curve is of utmost importance.

\section{PERSPECTIVES}

It is time to overcome the divide between fundamental research in neurosciences, increasing reliance on FNI and neurocomputational modeling which usually do not take into account the structural constraints, and the neurosurgical routine which should preserve the neural connectivity under penalty of no postoperative recovery, but which can also propose new cognitive models based upon direct observation of the connectome into the OR. Dual training for juniors, at the start of their courses, would enhance their chance to create reciprocal connections across basic and clinical neuroscience, and to develop more translational research in their daily practice dedicated to brain understanding and restoration.

\section{AUTHOR CONTRIBUTIONS}

The author confirms being the sole contributor of this work and has approved it for publication.

\section{REFERENCES}

1. Yeo BT, Krienen FM, Sepulcre J, Sabuncu MR, Lashkari D, Hollinshead $\mathrm{M}$, et al. The organization of the human cerebral cortex estimated by intrinsic functional connectivity. J Neurophysiol. (2011) 106:112565. doi: 10.1152/jn.00338.2011

2. Sporns O. The human connectome: Origins and challenges. Neuroimage. (2013) 80:53-61. doi: 10.1016/j.neuroimage.2013.03.023

3. Van Essen DC, Smith SM, Barch DM, Behrens TE, Yacoub E, Ugurbil K, et al. The WU-Minn human connectome project: an overview Neuroimage. (2013) 80:62-79. doi: 10.1016/j.neuroimage.2013.05.041

4. Glasser MF, Coalson TS, Robinson EC, Hacker CD, Harwell J, Yacoub E, et al. A multi-modal parcellation of human cerebral cortex. Nature. (2016) 536:171-8. doi: 10.1038/nature18933

5. Penfield W, Boldrey E. Somatic motor and sensory representation in the cerebral cortex of man as studied by electrical stimulation. Brain. (1937) 60:389. doi: 10.1093/brain/60.4.389

6. Ojemann GA. Brain organization for language from the perspective of electrical stimulation mapping. Behav Brain Sci. (1983) 6:189-206. doi: 10.1017/S0140525X00015491

7. Eickhoff SB, Yeo BTT, Genon S. Imaging-based parcellations of the human brain. Nat Rev Neurosci. (2018) 19:672-86. doi: 10.1038/s41583-018-0071-7 
8. Jeurissen B, Descoteaux M, Mori S, Leemans A. Diffusion MRI fiber tractography of the brain. NMR Biomed. (2019) 32:e3785. doi: 10.1002/nbm.3785

9. Duffau H. Stimulation mapping of myelinated tracts in awake patients. Brain Plast. (2016) 2:99-113. doi: 10.3233/BPL-160027

10. Duffau $H$. What direct electrostimulation of the brain taught us about the human connectome: a three-level model of neural disruption. Front Hum Neurosci. (2020) 14:315 doi: 10.3389/fnhum.2020.00315

11. Rech F, Herbet G, Moritz-Gasser S, Duffau H. Somatotopic organization of the white matter tracts underpinning motor control in humans: an electrical stimulation study. Brain Struct Funct. (2016) 221:374353. doi: 10.1007/s00429-015-1129-1

12. Duffau H, Moritz-Gasser S, Mandonnet E, A. reexamination of neural basis of language processing: proposal of a dynamic hodotopical model from data provided by brain stimulation mapping during picture naming. Brain Lang. (2014) 131:1-10. doi: 10.1016/j.bandl.2013.05.011

13. Zemmoura I, Herbet G, Moritz-Gasser S, Duffau H. New insights into the neural network mediating reading processes provided by cortico-subcortical electrical mapping. Hum Brain Mapp. (2015) 36:2215-30. doi: 10.1002/hbm.22766

14. Herbet G, Moritz-Gasser S, Duffau H. Direct evidence for the contributive role of the right inferior fronto-occipital fasciculus in non-verbal semantic cognition. Brain Struct Funct. (2017) 222:1597-610. doi: 10.1007/s00429-016-1294-x

15. Mandonnet E, Vincent M, Valero-Cabré A, Faque V, Barberis M, Bonnetblanc F, et al. Network-level causal analysis of set-shifting during trail making test part B: A multimodal analysis of a glioma surgery case. Cortex. (2020) 132:238-49. doi: 10.1016/j.cortex.2020.08.021

16. Ng S, Herbet G, Lemaitre AL, Moritz-Gasser S, Duffau H. Disrupting self-evaluative processing with electrostimulation mapping during awake brain surgery. Sci Rep. (2021) 11:9386. doi: 10.1038/s41598-021-8 8916-y

17. Herbet G, Duffau H. Awake craniotomy and bedside cognitive mapping in neurosurgery. In: Pearson CM, Ecklund-Johnson E, Gale SD, editors. Neurosurgical Neuropsychology. Chennai: Academic Press (2019). p. 113-38.

18. Roux A, Lemaitre AL, Deverdun J, Ng S, Duffau H, Herbet G. Combining electrostimulation with fiber tracking to stratify the inferior fronto-occipital fasciculus. Front Neurosci. (2021) 15:683348. doi: 10.3389/fnins.2021.683348 eCollection 2021

19. Duffau H. Stimulation mapping of white matter tracts to study brain functional connectivity. Nat Rev Neurol. (2015) 11:255-65. doi: 10.1038/nrneurol.2015.51

20. Herbet G, Duffau H. Revisiting the functional anatomy of the human brain: toward a meta-networking theory of cerebral functions. Physiol Rev. (2020) 100:1181-228. doi: 10.1152/physrev.00033.2019

21. Picart T, Herbet G, Moritz-Gasser S, Duffau H. Iterative surgical resections of diffuse glioma with awake mapping: how to deal with cortical plasticity and connectomal constraints? Neurosurgery. (2018) 85:10516. doi: $10.1093 /$ neuros/nyy218

22. Duffau H. Functional mapping before and after low-grade glioma surgery: a new way to decipher various spatiotemporal patterns of individual neuroplastic potential in brain tumor patients. Cancers. (2020) 12:2611. doi: 10.3390/cancers12092611

23. Duffau H. Lessons from brain mapping in surgery for low-grade glioma: insights into associations between tumour and brain plasticity. Lancet Neurol. (2005) 4:476-86. doi: 10.1016/S1474-4422(05)70140-X

24. Duffau $H$. The huge plastic potential of adult brain and the role of connectomics: new insights provided by serial mappings in glioma surgery. Cortex. (2014) 58:325-37. doi: 10.1016/j.cortex.2013.08.005

25. Herbet G, Maheu M, Costi E, Lafargue G, Duffau H. Mapping neuroplastic potential in brain-damaged patients. Brain. (2016) 139:829-44. doi: 10.1093/brain/awv394

26. Duffau $\mathrm{H}$. A two-level model of interindividual anatomofunctional variability of the brain and its implications for neurosurgery. Cortex. (2017) 86:30313. doi: 10.1016/j.cortex.2015.12.009

27. Ius T, Angelini E, Thiebaut de. Schotten M, Mandonnet E, Duffau H. Evidence for potentials and limitations of brain plasticity using an atlas of functional resectability of WHO grade II gliomas: towards a -minimal common brain. Neuroimage. (2011) 56:992-1000. doi: 10.1016/j.neuroimage.2011 03.022

28. Mandonnet E, Duffau H. Broca's area: why was neurosurgery neglected for so long when seeking to re-establish the scientific truth? Brain. (2021). doi: 10.1093/brain/awab195 [Epub ahead of print].

29. Henderson F, Abdullah KG, Verma R, Brem S. Tractography and the connectome in neurosurgical treatment of gliomas: the premise, the progress, and the potential. Neurosurg Focus. (2020) 48:E6. doi: 10.3171/2019.11.FOCUS19785

30. Conti Nibali M, Rossi M, Sciortino T, Riva M, Gay LG, Pessina $\mathrm{F}$, et al. Preoperative surgical planning of glioma: limitations and reliability of fMRI and DTI tractography. J Neurosurg Sci. (2019) 63:12734. doi: 10.23736/S0390-5616.18.04597-6

31. Krivosheya D, Prabhu SS. Combining functional studies with intraoperative MRI in glioma surgery. Neurosurg Clin N Am. (2017) 28:487-7. doi: 10.1016/j.nec.2017.05.004

32. Potgieser AR, Wagemakers $M$, van Hulzen AL, de Jong BM, Hoving EW, Groen RJ. The role of diffusion tensor imaging in brain tumor surgery: a review of the literature. Clin Neurol Neurosurg. (2014) 124:518. doi: 10.1016/j.clineuro.2014.06.009

33. Pujol S, Wells W, Pierpaoli C, Brun C, Gee J, Cheng G, et al. The DTI challenge: toward standardized evaluation of diffusion tensor imaging tractography for neurosurgery: the DTI challenge on tractography for neurosurgery. J Neuroimaging. (2015) 25:875-82. doi: 10.1111/jon.12283

34. Maier-Hein KH, Neher PF, Houde JC, Côté MA, Garyfallidis E, Zhong J, et al. The challenge of mapping the human connectome based on diffusion tractography. Nat Commun. (2017) 8:1349. doi: 10.1038/s41467-017-01285-x

35. Azad TD, Duffau H. Limitations of functional neuroimaging for patient selection and surgical planning in glioma surgery. Neurosurg Rev. (2020) 48:E12. doi: 10.3171/2019.11.FOCUS19769

36. Duffau $H$. The dangers of magnetic resonance imaging diffusion tensor tractography in brain surgery. World Neurosurg. (2014) 81:56-8. doi: 10.1016/j.wneu.2013.01.116

37. De Benedictis A, Nocerino E, Menna F, Remondino F, Barbareschi M, Rozzanigo U, et al. Photogrammetry of the human brain: a novel method for three-dimensional quantitative exploration of the structural connectivity in neurosurgery and neurosciences. World Neurosurg. (2018) 115:e27991. doi: 10.1016/j.wneu.2018.04.036

38. Panesar SS, Belo JTA, Yeh FC, Fernandez-Miranda JC. Structure, asymmetry, and connectivity of the human temporo-parietal aslant and vertical occipital fasciculi. Brain Struct Funct. (2019) 224:907-23. doi: 10.1007/s00429-018-1812-0

39. de Witt Hamer PC, Gil Robles S, Zwinderman A, Duffau H, Berger MS. Impact of intraoperative stimulation brain mapping on glioma surgery outcome: a meta-analysis. J Clin Oncol. (2012) 30:2559-65. doi: 10.1200/JCO.2011.38.4818

40. Duffau H. New philosophy, clinical pearls and methods for intraoperative cognition mapping and monitoring "à la carte" in brain tumor patients. Neurosurgery. (2021) 88:919-30. doi: 10.1093/neuros/nyaa363

41. Ferracci FX, Duffau H. Improving surgical outcome for gliomas with intraoperative mapping. Expert Rev Neurother. (2018) 18:333-41. doi: 10.1080/14737175.2018.1451329

42. Hervey-Jumper SL Li J, Lau D, Molinaro AM, Perry DW, Meng L, et al. Awake craniotomy to maximize glioma resection: methods and technical nuances over a 27-year period. J Neurosurg. (2015) 123:32539. doi: 10.3171/2014.10.JNS141520

43. Boetto J, Bertram L, Moulinié G, Herbet G, Moritz-Gasser S, Duffau H. Low rate of intraoperative seizures during awake craniotomy in a prospective cohort with 374 supratentorial brain lesions: electrocorticography is not mandatory. World Neurosurg. (2015) 84:1838-44. doi: 10.1016/j.wneu.2015.07.075

44. Bu LH, Zhang J, Lu JF, Wu JS. Glioma surgery with awake language mapping versus generalized anesthesia: a systematic review. Neurosurg Rev. (2020). doi: 10.1007/s10143-020-01418-9 [Epub ahead of print].

45. Mandonnet E, De Witt Hamer P, Poisson I, Whittle I, Bernat AL, Bresson D, et al. Initial experience using awake surgery for glioma: oncological, functional, and employment outcomes in a consecutive series of 25 cases. Neurosurgery. (2015) 76:382-9. doi: 10.1227/NEU.0000000000000644 
46. Lemaitre AL, Herbet G, Duffau H, Lafargue G. Preserved metacognitive ability despite unilateral or bilateral anterior prefrontal resection. Brain Cogn. (2018) 120:48-57. doi: 10.1016/j.bandc.2017.10.004

47. Barzilai O, BenMoshe S, Sitt R, Sela G, Shofty B, Ram Z. Improvement in cognitive function after surgery for low-grade glioma. J Neurosurg. (2019) 130:426-34. doi: 10.3171/2017.9.JNS17658

48. Ng S, Herbet G, Lemaitre AL, Cochereau J, Moritz-Gasser S, Duffau H Neuropsychological assessments before and after awake surgery for incidental low-grade gliomas. J Neurosurg. (2020) 1-10. doi: 10.3171/2020.7.JNS201507 [Epub ahead of print].

49. Ng S, Herbet G, Moritz-Gasser S, Duffau H. Return to work following surgery for incidental diffuse low-grade glioma: a prospective series with 74 patients. Neurosurgery. (2020) 87:720-9. doi: 10.1093/neuros/nyz513

50. Chang EF, Clark A, Smith JS, Polley MY, Chang SM, Barbaro NM, et al. Functional mapping-guided resection of low-grade gliomas in eloquent areas of the brain: improvement of long-term survival. Clinical article. J Neurosurg. (2011) 114:566-73. doi: 10.3171/2010.6.JNS091246

51. Duffau H. Long-term outcomes after supratotal resection of diffuse low-grade gliomas: a consecutive series with 11-year follow-up. Acta Neurochir (Wien). (2016) 158:51-8. doi: 10.1007/s00701-015-2621-3

52. Rossi M, Gay L, Ambrogi F, Nibali MC, Sciortino T, Puglisi G, et al. Association of supratotal resection with progression-free survival, malignant transformation, and overall survival in lower-grade gliomas. Neuro Oncol. (2021) 23:812-26. doi: 10.1093/neuonc/noaa225

53. Zigiotto L, Annicchiarico L, Corsino F, Vitali L, Falchi R, Dalpiaz C, et al. Effects of supra-total resection in neurocognitive and oncological outcome of high-grade gliomas comparing asleep and awake surgery. J Neurooncol. (2020) 148:97-108. doi: 10.1007/s11060-020-03494-9

54. Sarubbo S, De Benedictis A, Merler S, Mandonnet E, Balbi S, Granieri E, et al. Towards a functional atlas of human white matter. Hum Brain Mapp. (2015) 36:3117-36. doi: 10.1002/hbm.22832

55. Sarubbo S, Tate M, De Benedictis A, Merler S, Moritz-Gasser S, Herbet G, et al. Mapping critical cortical hubs and white matter pathways by direct electrical stimulation: an original functional atlas of the human brain. Neuroimage. (2020) 205:116237. doi: 10.1016/j.neuroimage.2019.116237

56. Sarubbo S, Tate M, De Benedictis A, Merler S, Moritz-Gasser S, Herbet G, et al. A normalized dataset of 1821 cortical and subcortical functional responses collected during direct electrical stimulation in patients undergoing awake brain surgery. Data Brief. (2019) 28:104892. doi: 10.1016/j.dib.2019.104892

57. Sarubbo S, Annicchiarico L, Corsini F, Zigiotto L, Herbet G, Moritz-Gasser S, et al. Planning brain tumor resection using a probabilistic atlas of cortical and subcortical structures critical for functional processing: a proof of concept. Oper Neurosurg. (2021) 20:E175-183. doi: 10.1093/ons/opaa396

58. Mandonnet E, Jbabdi S, Taillandier L, Galanaud D, Benali H, Capelle L, et al. Preoperative estimation of residual volume for WHO grade II glioma resected with intraoperative functional mapping. Neuro Oncol. (2007) 9:639. doi: 10.1215/15228517-2006-015
59. Rahimpour S, Haglund MM, Friedman AH, Duffau H. History of awake mapping and speech and language localization: from modules to networks. Neurosurg Focus. (2019) 47:E4. doi: 10.3171/2019.7.FOCUS 19347

60. Maesawa S, Nakatsubo D, Fujii M, Iijima K, Kato S, Ishizaki T. et al. Application of awake surgery for epilepsy in clinical practice. Neurol Med Chir. (2018) 58:442-52. doi: 10.2176/nmc.oa.2018-0122

61. Vigren $P$, Eriksson $M$, Duffau $H$, Wretman A, Lindehammar $H$, Milos P, et al. Experiences of awake surgery in non-tumoural epilepsy in eloquent localizations. Clin Neurol Neurosurg. (2020) 199:106251. doi: 10.1016/j.clineuro.2020.106251

62. Bourdillon P, Apra C, Guénot M, Duffau H. Similarities and differences in neuroplasticity mechanisms between brain gliomas and nonlesional epilepsy. Epilepsia. (2017) 58:2038-47. doi: 10.1111/epi.13935

63. Mandonnet E, Martino J, Sarubbo S, Corrivetti F, Bouazza S, Bresson D, et al. Neuronavigated fiber dissection with pial preservation: laboratory model to simulate opercular approaches to insular tumors. World Neurosurg. (2017) 98:239-42. doi: 10.1016/j.wneu.2016.10.020

64. Brown DA, Hanalioglu S, Chaichana K, Duffau H. Transcorticosubcortical approach for left posterior mediobasal temporal region gliomas: a case series and anatomic review of relevant white matter tracts. World Neurosurg. (2020) 139:e737-47. doi: 10.1016/j.wneu.2020. 04.147

65. Matsuda R, Coello AF, De Benedictis A, Martinoni M, Duffau H. Awake mapping for resection of cavernous angioma and surrounding gliosis in the left dominant hemisphere: surgical technique and functional results: clinical article. J Neurosurg. (2012) 117:1076-81. doi: 10.3171/2012.9.JNS 12662

66. Gayoso S, Perez-Borreda P, Gutierrez A, García-Porrero JA, Marco de. Lucas E, Martino J. Ventral precentral fiber intersection area: a central hub in the connectivity of perisylvian associative tracts. Oper Neurosurg. (2019) 17:182-92. doi: 10.1093/ons/opy331

67. Martino J, da Silva-Freitas R, Caballero H, Marco de Lucas E, GarcíaPorrero JA, Vázquez-Barquero A. Fiber dissection and diffusion tensor imaging tractography study of the temporoparietal fiber intersection area. Neurosurgery. (2013) 72(1 Suppl. Operative):8797. doi: 10.1227/NEU.0b013e318274294b

Conflict of Interest: The author declares that the research was conducted in the absence of any commercial or financial relationships that could be construed as a potential conflict of interest.

Copyright (C) 2021 Duffau. This is an open-access article distributed under the terms of the Creative Commons Attribution License (CC BY). The use, distribution or reproduction in other forums is permitted, provided the original author(s) and the copyright owner(s) are credited and that the original publication in this journal is cited, in accordance with accepted academic practice. No use, distribution or reproduction is permitted which does not comply with these terms. 